\title{
Heterotic estimation and adaptability of tomato hybrids for fruit yield and its related traits in Pakistan
}

\author{
Ahsan Javed ${ }^{1}$, Atif Akram², Muhammad Ijaz Tabassum ${ }^{1}$, Nadeem Ahmad ${ }^{1}$, Muhammad \\ Sarwar $^{1}$, Muhammad Jawaad Atif ${ }^{2}$ \\ ${ }^{1}$ Wheat Research Institute, Faisalabad, Pakistan. E-mail: ahsanwri@gmail.com, drijaztabassum07@gmail.com, \\ nadeemwri@gmail.com, sarwarvri18@gmail.com \\ ${ }^{2}$ Pakistan Agricultural Research Council, Islamabad, Pakistan. E-mail: atifakram250@gmail.com, jawaadatif@gmail.com
}

Received: 03/08/2021; Accepted: 10/12/21

\section{ABSTRACT}

Here, we estimated heterosis for different yield-attributing traits and the adaptability of tomato hybrids. The study was conducted at the experimental site of the Vegetable Crops Program/HRI/NARC in Islamabad, Pakistan from 2018-2019. Six parental lines (Continental, Nagina, Naqeeb, Peto 86, Riograndi, and Roma) and nine $F_{1}$ hybrid combinations were evaluated. Data analysis revealed statistically significant differences $(\mathrm{P} \leq 0.01)$ for all traits. Hybrids Peto $86 \times$ Nagina and Riograndi $\times$ Roma were found suitable for early maturing hybrids due to desirable high negative heterosis values. Maximum positive heterosis was observed in hybrids Riograndi $\times$ Nagina for plant height, Naqeeb $\times$ Roma for number of clusters plant ${ }^{-1}$, Naqeeb $\times$ Continental for number of flowers and fruits cluster- $^{-1}$, fruit length and width, and single fruit weight, and Riograndi $\times$ Continental for yield. In conclusion, $\mathrm{F}_{1}$ hybrid Naqeeb $\times$ Continental performed best of all nine hybrids tested and shows promising potential for use in different breeding programs.

Keywords: Heterosis, Heterobeltiosis, Hybrid vigor.

\section{Estimativa heterótica e adaptabilidade de híbridos de tomate para produção de frutos e suas características relacionadas no Paquistão}

\section{RESUMO}

Aqui, nós estimamos a heterose para diferentes características de atributos de rendimento e a adaptabilidade de tomates híbridos. O estudo foi conduzido na área experimental do "Programa de Produção de Hortaliças/HRI/NARC, em Islamabad, Paquistão, nos anos de 2018 e 2019. Foram avaliadas seis linhagens parentais (Continental, Nagina, Naqeeb, Peto 86, Riograndi, e Roma) e nove combinações híbridas F1. A análise dos dados revelou diferenças estatisticamente significativas $(\mathrm{P} \leq 0.01)$ para todas as características. Os híbridos Peto $86 \times$ Nagina e Riograndi $\times$ Roma foram considerados adequados para a maturação precoce devido aos altos valores de heterose negativa desejáveis. A máxima heterose positiva foi observada no híbrido Riograndi $\times$ Nagina para altura das plantas, no híbrido Naqeeb $\times$ Roma para número de cachos por planta, no híbrido Naqeeb $\times$ Continental para número de flores e frutos por cachos, comprimento, largura e peso individual dos frutos, e no híbrido Riograndi $\times$ Continental para o rendimento (produtividade). Concluindo, a geração F1 do híbrido Naqeeb $\times$ Continental foi o melhor de todos os nove híbridos testados e tem potencial promissor para uso em diferentes programas de melhoramento.

Palavras-chave: Heterose, Heterobeltiose, Vigor híbrido. 


\section{Introduction}

Tomato (Solanum lycopersicum L., Solanaceae), one of the most important vegetable crops in the world, has a chromosome number of $2 n=24$ and is largely selfpollinated in nature. Tomato is grown in nearly all climate regions (temperate, tropical, and subtropical climates) of the world. However, lower yields have become an increasingly important issue as global tomato consumption increases. Tomato is preferred by consumers for its high nutritional value and can be used in diverse ways. In Pakistan, tomato is consumed on a daily basis cooked with meat, pulses, and vegetables, processed into ketchup, or used in fresh salads (Ramzan et al., 2014). Tomato is a rich source of lycopene and other antioxidants and its consumption has been linked to a reduction in the risk of cardiovascular diseases and osteoporosis and increased skin protection from ultraviolet light-induced damage.

A regular tomato variety yields on average approximately 16-25 tonnes/ha, whereas a hybrid one produces 60-80 tonnes/ha (Sunil et al., 2013). In Pakistan, tomato cultivated area and production were 16,930 ha and 147,572 tonnes for the Kharif season (i.e., summer crop) and 38,328 ha and 4,137,221 tonnes for the Rabi season (i.e., winter crop) in 2019 (GOP, 2019). Thus, tomato yields in Pakistan provide only a small contribution to global production. In addition, the shortage and unavailability of quality seeds, inconsistent production during the year, and poor selection of genotypes for different environmental conditions and stresses are the major contributing factors to declining tomato yields in Pakistan.

Tomato, being a self-pollinated crop, has enormous potential for heterosis breeding. Shull (1914) first used the term heterosis in plants to describe the superiority of an $F_{1}$ hybrid over both its parents in terms of yield or adaptive behavior. Hedrick and Booth (1907) were the first to study heterosis in tomato for different yieldenhancing traits and several others have found heterosis in various tomato genotypes (e.g., Bhatt et al., 1998, 2001). For example, Kumar et al. (2003) reported 60\% heterosis for tomato cross combinations in India.

Heterosis is an important indicator for greater vigor, earliness, and increased productivity in tomato and key to improving the yield quantity and quality of tomato crops (Tamta and Singh, 2017). Moreover, there is scope for commercial exploitation of heterosis in tomato crops because it offers many advantages for marketable fruit yield, and selection of suitable parents is paramount to the success of commercial systems.

Improvements in yield and its various contributing traits as well as earliness can be achieved by exploiting heterosis in tomato. The relative breeding potential of parents can be assessed through genetic analysis for identification of best combiners in crops, which ultimately helps to evolve a variety, either by exploiting $\mathrm{F}_{1}$ heterosis or through accumulation of fixable genes (Sulodhani Devi et al., 2005). Thus, this study aimed to determine the optimal degree of heterosis in parent selection or hybridization for development of cross combinations and to select a suitable breeding procedure for yield improvement in tomato.

\section{Material and Methods}

This study was conducted at the experimental site of the Vegetable Crops Program at the Horticultural Research Institute (HRI) of the National Agricultural Research Center (NARC) in Islamabad, Pakistan from 2018-2019. Hybrid combinations were produced in 2018 whereas evaluation of six parental lines (Continental, Nagina, Naqeeb, Peto 86, Riograndi, and Roma) and nine $\mathrm{F}_{1}$ hybrid combinations was performed in 2019 using a randomized complete block design (RCBD) with three replications. Plants were spaced $50 \mathrm{~cm}$ apart in rows that were $75 \mathrm{~cm}$ apart. All cultural and agronomic practices were applied as and when required during the growing season of the crop.

The following plant traits were evaluated: days to 50 $\%$ flowering, number of clusters plant ${ }^{-1}$, number of flowers cluster ${ }^{-1}$, number of fruits cluster ${ }^{-1}$, days to $50 \%$ maturity, plant height $(\mathrm{cm})$, fruit length and width $(\mathrm{cm})$, single fruit weight $(\mathrm{g})$, and yield $\left(\mathrm{kg} \mathrm{plant}^{-1}\right)$. Data were analyzed using analysis of variance (ANOVA) followed by comparisons of means at $1 \%$ significance as described by Steel et al. (1997). All analyses were performed using AGRI-STAT software (ICAR, Old Goa, Goa, India). The degree of mid and better parent heterosis was computed using the following formulas:

Heterosis:

$$
\begin{gathered}
\operatorname{MP}(\mathrm{t})=\mathrm{F}_{1}-\mathrm{MP} /(3 / 2 \mathrm{r}) \mathrm{MSE} \\
\operatorname{BP}(\mathrm{t})=\mathrm{F}_{1}-\mathrm{BP} /(2 / \mathrm{r}) \mathrm{MSE}
\end{gathered}
$$

Where $\mathrm{MP}=$ mid parent value, $\mathrm{BP}=$ better parent value, $F_{1}=$ mean of $F_{1}$ hybrid for a particular trait, and $\mathrm{MSE}=$ mean squared error.

\section{Heterobeltiosis:}

$$
\mathrm{BPH} \%=\left[\mathrm{F}_{1}-\mathrm{BP} / \mathrm{BP}\right] \times 100
$$

Where $\mathrm{BPH}=$ better parent heterosis value and $\mathrm{BP}=$ better parent value.

\section{Results and Discussion}

In this study, highly significant differences were detected among tomato genotypes for all plant traits. These significant differences among plant traits are indicative of genetic variability, which can play an important role in yield improvement of tomato in different breeding programs. The mean performance of six tomato parental lines and nine $F_{1}$ hybrids is shown in Table 1 . There is an tomato early flowering; 50\% flowering across genotypes varied from 63-71 days (Table 2) 
Table 1. Analysis of variance (ANOVA) for yield and yield-attributing traits of 15 tomato genotypes

\begin{tabular}{|c|c|c|c|c|c|c|c|c|c|c|c|}
\hline SOV & df & DFL & $\mathrm{DM}$ & $\mathrm{PH}(\mathrm{cm})$ & $\mathrm{NCP}$ & NFC & NFRC & $\mathrm{FL}(\mathrm{cm})$ & $\mathrm{FW}(\mathrm{cm})$ & AFW (g) & FYP \\
\hline Replication & 2 & 4.956 & 2.489 & 11.588 & 18.756 & 0.019 & 0.127 & 0.067 & 0.025 & 36.005 & 0.013 \\
\hline Treatment & 14 & $13.546^{* *}$ & $12.946^{* *}$ & $142.429 * *$ & $47.994 * *$ & $0.929 * *$ & $0.729 * *$ & $0.863 * *$ & $0.593 * *$ & $600.534 * *$ & $0.407 * *$ \\
\hline Error & 28 & 0.408 & 1.537 & 3.048 & 5.137 & 0.102 & 0.086 & 0.115 & 0.031 & 20.303 & 0.005 \\
\hline CV (\%) & & 0.97 & 1.09 & 2.38 & 8.24 & 5.89 & 8.07 & 5.71 & 3.48 & 6.31 & 5.56 \\
\hline
\end{tabular}

**Significant at $\mathrm{P} \leq 0.01 ; \mathrm{AFW}=$ average fruit weight, $\mathrm{df}=$ degrees of freedom, $\mathrm{DFL}=$ days to $50 \%$ flowering, $\mathrm{DM}=$ days to $50 \%$ maturity, $\mathrm{FL}=$ fruit length, $\mathrm{FW}=$ fruit width, $\mathrm{FYP}=$

fruit yield/plant, $\mathrm{NCP}=$ number of clusters/plant, $\mathrm{NFC}=$ number of flowers/cluster, $\mathrm{NFRC}=$ number of fruits/cluster, and $\mathrm{PH}=\mathrm{plant}$ height.

Table 2. Mean values for yield and yield-attributing traits of 15 tomato genotypes.

\begin{tabular}{|c|c|c|c|c|c|c|c|c|c|c|}
\hline Genotype & $\begin{array}{c}\text { Days to } \\
50 \% \\
\text { flowering }\end{array}$ & $\begin{array}{c}\text { Days to } 50 \% \\
\text { maturity }\end{array}$ & Plant height $(\mathrm{cm})$ & $\begin{array}{c}\text { No. of } \\
\text { clusters/plant }\end{array}$ & $\begin{array}{c}\text { No. of } \\
\text { flowers/cluster }\end{array}$ & $\begin{array}{l}\text { No. of } \\
\text { fruits/cluster }\end{array}$ & $\begin{array}{l}\text { Fruit length } \\
\qquad(\mathrm{cm})\end{array}$ & $\begin{array}{l}\text { Fruit width } \\
\quad(\mathrm{cm})\end{array}$ & $\begin{array}{l}\text { Average } \\
\text { fruit } \\
\text { weight }(g)\end{array}$ & $\begin{array}{c}\text { Yield } \\
\text { (kg/plant) }\end{array}$ \\
\hline Roma & $67 \mathrm{C}$ & $117 \mathrm{AB}$ & $73.33 \mathrm{CDE}$ & $25 \mathrm{DEF}$ & $5.7 \mathrm{BC}$ & $3.3 \mathrm{EFG}$ & $6.7 \mathrm{AB}$ & $4.88 \mathrm{D}$ & $58.17 \mathrm{DE}$ & $0.51 \mathrm{EF}$ \\
\hline Nagina & $65 \mathrm{D}$ & $114 \mathrm{CD}$ & $70.76 \mathrm{EFGH}$ & $27 \mathrm{CDE}$ & $5.1 \mathrm{DE}$ & $3.9 \mathrm{CD}$ & 5.61 EFG & $4.68 \mathrm{D}$ & $58 \mathrm{DE}$ & $0.57 \mathrm{E}$ \\
\hline Continental & $63 \mathrm{EF}$ & $112 \mathrm{DEF}$ & $92 \mathrm{~A}$ & $26 \mathrm{DEF}$ & $5.3 \mathrm{CDE}$ & $3.3 \mathrm{EFG}$ & $5.01 \mathrm{H}$ & $6.14 \mathrm{~A}$ & $94 \mathrm{~A}$ & $0.46 \mathrm{~F}$ \\
\hline Peto 86 & $64 \mathrm{DE}$ & $113 \mathrm{CDE}$ & $68.66 \mathrm{HI}$ & $26 \mathrm{DEF}$ & $4 \mathrm{~F}$ & $3.3 \mathrm{EFG}$ & $5.34 \mathrm{FGH}$ & $4.76 \mathrm{D}$ & $62 \mathrm{D}$ & $0.47 \mathrm{~F}$ \\
\hline Riograndi & $65 \mathrm{D}$ & $114 \mathrm{CD}$ & $70.56 \mathrm{EFGH}$ & $26 \mathrm{DEF}$ & 5.3 CDE & $3.2 \mathrm{EFG}$ & $6.84 \mathrm{~A}$ & $5.26 \mathrm{BC}$ & $90 \mathrm{AB}$ & $0.57 \mathrm{E}$ \\
\hline Naqeeb & $69 \mathrm{~B}$ & $118 \mathrm{~A}$ & 69.86 FGH & $21 \mathrm{G}$ & $5 \mathrm{EF}$ & $3 \mathrm{G}$ & $5.95 \mathrm{DE}$ & $4.77 \mathrm{D}$ & $54 \mathrm{EF}$ & $0.52 \mathrm{EF}$ \\
\hline Peto $86 \times$ Roma & $64 \mathrm{DE}$ & $111 \mathrm{EF}$ & $68.76 \mathrm{GHI}$ & $28 \mathrm{BCDE}$ & $5 \mathrm{EF}$ & $3.5 \mathrm{DEF}$ & 5.86 DEF & $4.77 \mathrm{D}$ & $90.13 \mathrm{AB}$ & $1.01 \mathrm{D}$ \\
\hline Peto $86 \times$ Nagina & $63 \mathrm{~F}$ & $110 \mathrm{~F}$ & $71.56 \mathrm{DEFGH}$ & $29 \mathrm{BCD}$ & 5.6 BCD & $3.9 \mathrm{CD}$ & $5.74 \mathrm{DEF}$ & $4.71 \mathrm{D}$ & $71.37 \mathrm{C}$ & $1.19 \mathrm{C}$ \\
\hline Peto $86 \times$ Continental & $65 \mathrm{D}$ & $113 \mathrm{CDE}$ & $74 \mathrm{CD}$ & $25 \mathrm{EFG}$ & $5 \mathrm{EF}$ & $3.2 \mathrm{EFG}$ & $5.98 \mathrm{DE}$ & $5.19 \mathrm{C}$ & $73.87 \mathrm{C}$ & $1.16 \mathrm{C}$ \\
\hline Riograndi $\times$ Roma & $65 \mathrm{D}$ & $111 \mathrm{EF}$ & $71.76 \mathrm{CDEF}$ & $23 \mathrm{FG}$ & $5.2 \mathrm{CDE}$ & 3.7 CDE & $5.65 \mathrm{EFG}$ & $4.69 \mathrm{D}$ & $60.37 \mathrm{DE}$ & $1.01 \mathrm{D}$ \\
\hline Riograndi $\times$ Nagina & $65 \mathrm{D}$ & $112 \mathrm{DEF}$ & $86 \mathrm{~B}$ & $31 \mathrm{~B}$ & $6 \mathrm{AB}$ & $5 \mathrm{~A}$ & $6.03 \mathrm{CDE}$ & $5.5 \mathrm{~B}$ & $76.7 \mathrm{C}$ & $1.43 \mathrm{~B}$ \\
\hline Riograndi $\times$ Continental & $68 \mathrm{BC}$ & $114 \mathrm{CD}$ & 71.66 DEFG & $30 \mathrm{BC}$ & $5.2 \mathrm{CDE}$ & $4 \mathrm{BC}$ & $6.58 \mathrm{ABC}$ & $4.71 \mathrm{D}$ & $86.43 \mathrm{~B}$ & $1.58 \mathrm{~A}$ \\
\hline Naqeeb $\times$ Roma & $67 \mathrm{C}$ & $113 \mathrm{CDE}$ & $70.1 \mathrm{FGH}$ & $38 \mathrm{~A}$ & $5.2 \mathrm{CDE}$ & $3.1 \mathrm{FG}$ & $6.27 \mathrm{BCD}$ & $5.42 \mathrm{BC}$ & $74.4 \mathrm{C}$ & $1.02 \mathrm{D}$ \\
\hline Naqeeb $\times$ Nagina & $68 \mathrm{BC}$ & $114 \mathrm{C}$ & $66.66 \mathrm{I}$ & $26 \mathrm{DEF}$ & $6 \mathrm{AB}$ & 4.1 $\mathrm{ABC}$ & 6.17BCDE & $5.54 \mathrm{~B}$ & $72.07 \mathrm{C}$ & $1.01 \mathrm{D}$ \\
\hline Naqeeb $\times$ Continental & $71 \mathrm{~A}$ & $115 \mathrm{BC}$ & $74.66 \mathrm{C}$ & $30 \mathrm{BC}$ & $7 \mathrm{~A}$ & $4.5 \mathrm{AB}$ & $5.15 \mathrm{GH}$ & $4.65 \mathrm{D}$ & $50 \mathrm{~F}$ & $1.13 \mathrm{C}$ \\
\hline Range & $63-71$ & $110-118$ & $66.66-92$ & $21-38$ & $7-4$ & $3-5$ & $5.01-6.84$ & $4.65-6.14$ & $50-94$ & $0.46-1.58$ \\
\hline
\end{tabular}


The degree of heterosis for tomato genotypes ranged from $-1.96 \%$ to $-3.34 \%$ over mid parent, $-2.91 \%$ to $-4.46 \%$ over better parent, and $-3.57 \%$ to $-4.08 \%$ over standard parent. Of the nine $F_{1}$ hybrids, four cross combinations exhibited negative heterosis values over mid parent, four over better parent, and two over standard parent. The Peto $86 \times$ Nagina cross showed maximum negative heterosis over mid parent $(-3.34 \%)$, better parent $(-4.08 \%)$, and standard parent $(-4.08 \%)$ for days to $50 \%$ maturity (Table 3a). Similarly, AlamPatwary et al. (2013) and Chauhan et al. (2014) also found earliness in heterotic tomato combinations.

Early production may extend the supply season providing market opportunities, which ultimately helps growers receive the remunerative price. Mean days to $50 \%$ maturity across genotypes ranged from 110-118 days (Table 2). The degree of heterosis across genotypes ranged from $-1.75 \%$ to $-3.61 \%$ over mid parent, $-2.54 \%$ to $-4.84 \%$ over better parent, and $-2.34 \%$ to $-3.22 \%$ over standard parent. Of the nine $\mathrm{F}_{1}$ hybrids, five cross combinations showed negative heterosis over mid parent, six over better parent, and three over standard parent. The Riograndi $\times$ Roma cross showed maximum negative heterosis over mid $(-3.62 \%)$ and better parent $(-4.84 \%)$, whereas the Peto $86 \times$ Nagina combination exhibited maximum negative heterosis over standard parent $(-3.22 \%$ ) for days to $50 \%$ maturity (Table 3a). Similarly, Singh et al. (2012) also found early maturity in heterotic tomato combinations.

Increased focus on reproductive growth is required for improving fruit production. Mean plant height across genotypes varied from 66.66 to $92.0 \mathrm{~cm}$ (Table 2). The degree of heterosis across genotypes ranged from 5.19$21.7 \%$ over mid parent, $5.79-21.53 \%$ over better parent, and $5.53-21.87 \%$ over standard parent. Of the nine $\mathrm{F}_{1}$ hybrids, three crosses each showed maximum positive heterosis over mid, better, and standard parent, respectively. In addition, the Riograndi $\times$ Nagina cross showed maximum positive heterosis values over mid parent $(21.7 \%)$, better parent $(21.53 \%)$, and standard parent $(21.87 \%)$ for plant height (Table 3a). Rai et al. (2003) also reported a similar increase in plant height of hybrids over parents in tomato.

Mean number of clusters plant ${ }^{-1}$ across genotypes varied from 21 to 38 (Table 2). The degree of heterosis across genotypes ranged from $16.25-61.7 \%$ over mid parent, $14.81-48.05 \%$ over better parent, and 16.46$44.3 \%$ over standard parent. Of the nine $F_{1}$ hybrids, four crosses each showed positive heterosis values over mid, better, and standard parent, respectively. The Naqeeb $\times$ Roma combination exhibited maximum positive heterosis over mid parent $(61.7 \%)$, better parent $(48.05 \%)$, and standard parent $(44.3 \%)$ across the nine $F_{1}$ hybrid combinations (Table 3a). These results are consistent with the findings of Garg and Cheema (2010).
Mean number of flowers cluster ${ }^{-1}$ across genotypes ranged from four to seven (Table 2). The degree of heterosis across genotypes varied from 17.24-29.87\% over mid parent, $11.63-25.0 \%$ over better parent, and $15.72-25.79 \%$ over standard parent. Of the nine $F_{1}$ hybrids, four cross combinations each exhibited positive heterosis values over mid and better parent and three over standard parent. The Naqeeb $\times$ Continental cross showed maximum positive heterosis over mid parent $(29.87 \%)$, better parent $(25.0 \%)$, and standard parent (25.79\%) for number of flowers cluster ${ }^{-1}$ (Table $\left.3 b\right)$.

Fruit cluster in a plant are important for more fruit yield. Mean number of fruits cluster $^{-1}$ across genotypes ranged from 3-5 (Table 2). The degree of heterosis across genotypes varied from 13.4-41.05\% over mid parent, $17.24-34.0 \%$ over better parent, and $22.92-41.67 \%$ over standard parent. Of the nine $F_{1}$ hybrids, five cross combinations showed positive heterosis over mid parent, three over better parent, and five over standard parent. The Naqeeb $\times$ Continental cross exhibited positive heterosis over mid parent $(41.05 \%)$ and better parent $(34.0 \%)$, whereas the Riograndi $\times$ Nagina combination showed maximum positive heterosis $(41.67 \%)$ over standard parent for number of fruits cluster ${ }^{-1}$ (Table $3 b$ ). Gul et al. (2010) also found significant heterosis for fruits per cluster in tomato.

Mean fruit length across genotypes ranged from 5.01 to $6.84 \mathrm{~cm}$ (Table 2). The degree of heterosis across genotypes varied from 11.04-15.46\% over mid parent, $3.74-11.83 \%$ over better parent, and $11.73-24.61 \%$ over standard parent. Of the nine $F_{1}$ hybrids, two crosses exhibited positive heterosis over mid parent, two over better parent, and six over standard parent. The Peto $86 \times$ Continental cross showed maximum positive heterosis over mid parent $(15.46 \%)$ and better parent $(11.83 \%)$, whereas the Naqeeb $\times$ Continental cross combination showed maximum positive heterosis (24.61\%) over standard parent (Table 3b). Chattopadhyay and Paul (2012) also found significant heterosis for fruit length in tomato.

Mean fruit width across genotypes ranged from $4.65-6.14 \mathrm{~cm}$ (Table 2). The degree of heterosis across genotypes varied from $10.69-17.37 \%$ over mid parent, $10.99-16.25 \%$ over better parent, and 10.35-11.49\% over standard parent. Of the nine $\mathrm{F}_{1}$ hybrids, three crosses showed positive heterosis over mid parent, two over better parent, and four over standard parent. The Naqeeb $\times$ Nagina cross showed positive heterosis over mid parent (17.37\%) and better parent (16.25\%), whereas the Naqeeb $\times$ Continental combination exhibited maximum positive heterosis $(11.49 \%)$ over standard parent for fruit width (Table 3c). These results are consistent with the findings of Dev et al. (1994) for fruit width in heterotic tomato combinations. 
Table 3(a). Heterotic performance of nine tomato hybrid combinations over mid, better, and standard parents.

\begin{tabular}{|c|c|c|c|c|c|c|c|c|c|c|c|c|}
\hline \multirow{2}{*}{ Cross } & \multicolumn{3}{|c|}{ Days to $50 \%$ flowering } & \multicolumn{3}{|c|}{ Days to $50 \%$ maturity } & \multicolumn{3}{|c|}{ Plant height $(\mathrm{cm})$} & \multicolumn{3}{|c|}{ No. of clusters/plant } \\
\hline & $\mathrm{MPH}$ & $\mathrm{BPH}$ & SPH & $\mathrm{MPH}$ & $\mathrm{BPH}$ & SPH & $\mathrm{MPH}$ & $\mathrm{BPH}$ & SPH & $\mathrm{MPH}$ & $\mathrm{BPH}$ & SPH \\
\hline Peto $86 \times$ Roma & $-2.28 * *$ & $-4.46^{* *}$ & $-1.53 \mathrm{~ns}$ & $-3.19 * *$ & $-4.8 * *$ & $-2.34 *$ & -3.15 & $-6.23 * *$ & $-2.55 \mathrm{~ns}$ & 7.69 & 6.33 & $6.33 \mathrm{~ns}$ \\
\hline Peto $86 \times$ Nagina & $-3.34 * *$ & $-4.08 * *$ & $-4.08 * *$ & $-2.79 * *$ & $-3.22 * *$ & $-3.22 * *$ & 2.65 & 1.13 & $1.42 \mathrm{~ns}$ & 8.75 & 7.41 & $10.13 \mathrm{~ns}$ \\
\hline Peto $86 \times$ Continental & $2.35 * *$ & 1.55 & $-0.0 \mathrm{~ns}$ & 0.44 & 0.93 & $-0.88 \mathrm{~ns}$ & $-8.21 * *$ & $-20.06^{* *}$ & $4.87 *$ & -4.46 & -5.06 & $-5.06 \mathrm{~ns}$ \\
\hline Riograndi $\times$ Roma & $-2.01 * *$ & $-3.47 * *$ & $-0.51 \mathrm{~ns}$ & $-3.61 * *$ & $-4.84 * *$ & $-2.34 *$ & -0.25 & -2.14 & $1.7 \mathrm{~ns}$ & $-12.82 *$ & -13.92 & $13.92 \mathrm{~ns}$ \\
\hline Riograndi $\times$ Nagina & 0.35 & 0.61 & $-0.0 \mathrm{~ns}$ & $-1.75^{*}$ & -1.75 & $-1.75 \mathrm{~ns}$ & $21.7 * *$ & $21.53 * *$ & $21.87 * *$ & $16.25^{*}$ & $14.81 *$ & $17.72 *$ \\
\hline Riograndi $\times$ Continental & $5.18 * *$ & $3.57 * *$ & $3.57 * *$ & 0.59 & -0.29 & $-0.29 \mathrm{~ns}$ & $-12.14 * *$ & $-22.58 * *$ & $1.56 \mathrm{~ns}$ & $17.2 * *$ & $16.46^{*}$ & $16.46^{*}$ \\
\hline Naqeeb $\times$ Roma & $-1.96 * *$ & $-2.91 * *$ & $2.04^{*}$ & $-3.55^{* *}$ & $-3.95 * *$ & $-0.58 \mathrm{~ns}$ & -2.09 & $-4.41^{*}$ & $-0.66 \mathrm{~ns}$ & $61.7 * *$ & $48.05 * *$ & $44.3 * *$ \\
\hline Naqeeb $\times$ Nagina & 1.0 & -1.46 & & -1.44 & $-3.11 * *$ & $0.29 \mathrm{~ns}$ & $5.19 * *$ & $5.79 * *$ & $5.53 *$ & 4.83 & -6.17 & $-3.8 \mathrm{~ns}$ \\
\hline Naqeeb $\times$ Continental & $7.07 * *$ & $2.91 * *$ & $8.16^{* *}$ & 0.77 & $-2.54 * *$ & $0.88 \mathrm{~ns}$ & $8.06^{* *}$ & $19.34 * *$ & $5.81 * *$ & $28.17 * *$ & $16.67 *$ & $15.19^{*}$ \\
\hline
\end{tabular}

$\mathrm{MPH}=$ mid parent heterosis, $\mathrm{BPH}=$ better parent heterosis, and $\mathrm{SPH}=$ standard parent heterosis.

Table 3(b). Heterotic performance of nine tomato hybrid combinations over mid, better, and standard parents.

\begin{tabular}{|c|c|c|c|c|c|c|c|c|c|}
\hline \multirow{2}{*}{ Cross } & \multicolumn{3}{|c|}{ No. of flowers/cluster } & \multicolumn{3}{|c|}{ No. of fruits/cluster } & \multicolumn{3}{|c|}{ Fruit length $(\mathrm{cm})$} \\
\hline & MPH & $\mathrm{BPH}$ & SPH & MPH & $\mathrm{BPH}$ & SPH & $\mathrm{MPH}$ & $\mathrm{BPH}$ & SPH \\
\hline Peto $86 \times$ Roma & -1.3 & $11.63^{*}$ & $-4.4 \mathrm{~ns}$ & 7.61 & 7.07 & $10.42 \mathrm{~ns}$ & -2.6 & $-12.44 * *$ & $14.25^{* *}$ \\
\hline Peto $86 \times$ Nagina & $17.24 * *$ & 10.39 & $6.92 \mathrm{~ns}$ & 9.77 & 1.72 & $22.92 * *$ & 4.75 & 2.25 & $16.08^{* *}$ \\
\hline Peto $86 \times$ Continental & -0.68 & -8.13 & $-7.55 \mathrm{~ns}$ & -3.52 & -4 & $-0.0 \mathrm{~ns}$ & $15.46^{* *}$ & $11.83 *$ & $12.6^{* *}$ \\
\hline Riograndi $\times$ Roma & -5.14 & -8.72 & $-1.26 \mathrm{~ns}$ & $13.4^{*}$ & 12.24 & $14.58 \mathrm{~ns}$ & $-16.47 * *$ & $-17.33^{* *}$ & $17.33^{* *}$ \\
\hline Riograndi $\times$ Nagina & $17.57 * *$ & $15.72 * *$ & $15.72 * *$ & $28.3^{* *}$ & $17.24 * *$ & $41.67 * *$ & -3.04 & $-11.73 * *$ & $11.73 * *$ \\
\hline Riograndi $\times$ Continental & -0.94 & -1.25 & $-0.63 \mathrm{~ns}$ & $23.47 * *$ & $21.0 * *$ & $26.04 * *$ & $11.04 *$ & -3.82 & $-3.82 \mathrm{~ns}$ \\
\hline Naqeeb $\times$ Roma & -1.25 & -8.14 & $-0.63 \mathrm{~ns}$ & 0.0 & -4.08 & $-2.08 \mathrm{~ns}$ & -0.79 & -6.35 & $-8.29 \mathrm{~ns}$ \\
\hline Naqeeb $\times$ Nagina & $21.85 * *$ & $19.48 * *$ & $15.72 * *$ & $19.42 * *$ & 6.03 & $28.12 * *$ & 6.73 & $3.74 *$ & $-9.79 *$ \\
\hline Naqeeb $\times$ Continental & $29.87 * *$ & $25.0^{* *}$ & $25.79 * *$ & $41.05 * *$ & $34.0 * *$ & $39.58 * *$ & -5.87 & 3.61 & $24.61 * *$ \\
\hline
\end{tabular}

$\mathrm{MPH}=$ mid parent heterosis, $\mathrm{BPH}=$ better parent heterosis, and $\mathrm{SPH}=$ standard parent heterosis.

Table 3(c). Heterotic performance of nine tomato hybrid combinations over mid, better, and standard parents.

\begin{tabular}{|c|c|c|c|c|c|c|c|c|c|}
\hline \multirow{2}{*}{ Cross } & \multicolumn{3}{|c|}{ Fruit width $(\mathrm{cm})$} & \multicolumn{3}{|c|}{ Average fruit weight (g) } & \multicolumn{3}{|c|}{ Yield (kg/plant) } \\
\hline & $\mathrm{MPH}$ & $\mathrm{BPH}$ & SPH & $\mathrm{MPH}$ & $\mathrm{BPH}$ & SPH & $\mathrm{MPH}$ & $\mathrm{BPH}$ & SPH \\
\hline Peto $86 \times$ Roma & -1.0 & -2.27 & $-9.27 * *$ & $50.0 * *$ & $45.38 * *$ & $0.15 \mathrm{~ns}$ & $106.12 * *$ & $99.34 * *$ & $78.24 * *$ \\
\hline Peto $86 \times$ Nagina & -0.22 & -1.07 & $10.51 * *$ & $18.94 * *$ & $15.11 *$ & $20.7 * *$ & $128.75^{* *}$ & $109.36^{* *}$ & $110.59 *$ \\
\hline Peto $86 \times$ Continental & $-4.76^{*}$ & $-15.5 * *$ & -1.29 & -5.3 & $-21.42 * *$ & $17.93 * *$ & $148.75^{* *}$ & $144.37 * *$ & $104.12 *$ \\
\hline Riograndi $\times$ Roma & $-7.58 * *$ & $-10.89 * *$ & $10.89 * *$ & $-18.52 * *$ & $-32.93 * *$ & $32.93 * *$ & $88.2 * *$ & $78.24 * *$ & $78.24 * *$ \\
\hline Riograndi $\times$ Nagina & $10.69 * *$ & 4.56 & $4.56 \mathrm{~ns}$ & -3.65 & $-14.78 * *$ & $14.78 * *$ & $152.2 * *$ & $151.46^{* *}$ & $152.94 *$ \\
\hline Riograndi $\times$ Continental & $-17.3 * *$ & $-23.25 * *$ & $10.35 * *$ & -6.05 & $-8.05 *$ & -3.96 & $208.79 * *$ & $178.82 * *$ & $178.82 *$ \\
\hline Naqeeb $\times$ Roma & $12.32 * *$ & $10.99 * *$ & $3.04 \mathrm{~ns}$ & $32.65 * *$ & $27.89 * *$ & $17.33 * *$ & $98.05 * *$ & $95.51 * *$ & $79.41 * *$ \\
\hline Naqeeb $\times$ Nagina & $17.37 * *$ & $16.25 * *$ & $5.37 \mathrm{~ns}$ & $28.69 * *$ & $24.25 * *$ & $19.93 * *$ & $85.32 * *$ & $77.19 * *$ & $78.24 * *$ \\
\hline Naqeeb $\times$ Continental & 1.09 & 3.25 & $11.49 * *$ & $-32.43 * *$ & -2.33 & $44.44 * *$ & $132.08 * *$ & $117.95 * *$ & $100.0 * *$ \\
\hline
\end{tabular}


Mean average fruit weight across genotypes ranged from 50 to $94 \mathrm{~g}$ (Table 2). The degree of heterosis across genotypes varied from $18.94-50.0 \%$ over mid parent, $15.11-45.38 \%$ over better parent, and 14.78 $44.44 \%$ over standard parent. Of the nine $\mathrm{F}_{1}$ hybrids, four cross combinations showed positive heterosis values over mid parent, four over better parent, and seven over standard parent.

The Peto $86 \times$ Roma combination showed maximum positive heterosis over mid parent $(50.0 \%)$ and better parent $(45.38 \%)$, whereas the Naqeeb $\times$ Continental cross showed maximum positive heterosis (44.44\%) over standard parent for average fruit weight ( $g$ ) (Table $3 c)$. These results are consistent with the findings of Joshi and Thakur (2003) for average fruit weight in heterotic tomato combinations.

Mean fruit yield per plant across parents and hybrid combinations ranged from $0.46-1.58 \mathrm{~kg}$ plant $^{-1}$ (Table 2 ). The degree of heterosis across genotypes varied from $85.32-208.79 \%$ over mid parent, $77.19-178.82 \%$ over better parent, and $78.24-178.82 \%$ over standard parent. The $\mathrm{F}_{1}$ hybrid Riograndi× Continental showed maximum positive heterosis values for mid parent (208.78\%), better parent (178.82\%), and standard parent $(178.82 \%)$ across the nine $F_{1}$ hybrid combinations (Table 3c). Ahmad et al. (2011) also found high heterosis for yield per plant in tomato.

\section{Conclusions}

The ultimate goal of a plant breeder - and a basic requirement of any breeding program - is to increase yield, which correlates directly or indirectly with other plant traits. Thus, it is evident from the data that Naqeeb was important in exploiting hybrid vigor among testers and Continental played a key role among lines by contributing to traits such as number of clusters plant ${ }^{-1}$, number of flowers and fruits cluster ${ }^{-1}$, fruit length, fruit width, and average fruit weight.

Based on mean performance and heterobeltiosis, hybrids Naqeeb $\times$ Continental, Peto $86 \times$ Nagina, and Riograndi $\times$ Nagina should be investigated further for developing high-yield, quality tomato varieties.

\section{Authors' Contribution}

Atif Akram has planned and conducted the research and performed write-up. Ahsan Javed has conducted the research and performed write-up. Muhammad Ijaz Tabassum has revised the research work. Nadeem Ahmad has assisted in write-up. Muhammad Sarwar has analyzed the data and Muhammad Jawaad Atif has assisted in data collection.

\section{Bibliographic References}

Ahmad, S, Quarmruzzaman, A.K.M, Islam M.R. 2011. Estimation of heterosis in tomato (Solanum lycopersicum L.). Bangladesh Journal of Agricultural Research. 36(3): 521- 527. DOI: https://doi.org/10.3329/bjar.v36i3.9280

Alam-Patwary M.M, Rahman, M.M, Ahmad, S, Miah M.A.K. Barua, H. 2013. Study of heterosis in heat tolerant tomato (Solanum lycopersicum) during summer. Bangladesh Journal of Agricultural Research.38(3): 531-544. DOI: https:// doi:10.3329/BJAR.V38I3.16980

Bhatt, R.P, Biswas, V.R., Pandey, H.K., Verma, G.S., Narendra, K. 1998. Heterosis for vitamin C in tomato (Lycopersicon esculentum Mill). Indian Journal of Agricultural Sciences. 68: (1)176-178.

Bhatt, R.P, Biswas, V.R., Kumar, N. 2001. Heterosis, combining ability, genetics for vitamin $\mathrm{C}$, total soluble solids and yield in tomato (Lycopersicon esculentum Mill) at $1700 \mathrm{~m}$ altitude. Journal of Agricultural Science.137: 71-75. DOI: https://doi.org/10.1017/S0021859601008838

Chattopadhyay, A., Paul, A. 2012. Studies on heterosis for different fruit quality parameters in tomato. International Journal of Agriculture Environment Biotechnology.5(4): 405-410.

Chauhan, V.B.S, RajKumar Behera, T.K., Yadav, R.K. 2014. Studies on heterosis for yield and its attributing traits in tomato (Solanum lycopersicum L.). International Journal of Agriculture, environment \& biotechnology. 7(1):95-100.

Dev, H, Rattan, R.S, Thakur, M.C. 1994. Heterosis in tomato. The Horticulture Journal.7 (2): 125-132.

Garg, N., Cheema, D.S. 2010. Seeds of tomato (Solanum lycopersicum L.) hybrids incorporating rin, nor, or alc alleles exhibit heterosis for yield and quality traits. Journal of New Seeds.11:250-261. DOI: https://doi:10.1080/1522886X.2010 499323

Gul, R, Rahman, H, Khalil, I.H., Shah, S.M.A, Ghafoor, A. 2010. Heterosis for flower and fruit traits in tomato (Lycopersicum esculentum Mill.) African Journal of Biotechnology.9(27):41444151. http://www.academicjournals.org/AJB

GOP. GOVERNMENT OF PAKISTAN. 2019. Fruit, Vegetables and Condiments. Statistics of Pakistan. Ministry of National Food Security and Research (Economic Wing), Islamabad. http://www.mnfsr.gov.pk/pubDetails.aspx

Hedrick, U.P, Booth, N.O. 1907. Mendelian characters in tomato. Proceedings of American Society of Horticultural Sciences.5: (1)19-24.

Joshi, A. Thakur, M.C. 2003. Exploitation of heterosis for yield and yield contributing traits in tomato (Lycopersicon esculentum Mill.). Progressive Horticulture.3: (1) 564-68.

Kumar, S, Kumar, R, Kumar, S., Singh, M., Banerjee, M.K., Rai, M. 2003. Hybrid seed production of solanaceous vegetables: A Practical Manual, II VR Technical Bulletin. 9: (1)1-34.

Rai, M, Singh, A.K, Pan, R.S., Krishna, P.V.S.R. 2003. Combining ability of quality and yield in tomato (Lycopersicum esculentum Mill.). Vegetable Science. 30 (10): 21-24. 
Ramzan, A., Khan, T.N., Nawab, N.N., Hina, A., Noor, T., Jellani, G. 2014. Estimation of genetic components in $F_{1}$ hybrids and their parents in determinate tomato (Solanum lycopersicum L.). Journal of agricultural research.52(1): 6575.

Shull, G.H. 1914. Duplicate genes for capsule form in Bursa bursa-pastoris. Zeitscher. Induct. Abstamm. U. Verebungsl. 12:97-149.

Steel, R.G.D., Torrie, J.H., Deekey, D.A. 1997.Principles and procedures of statistics: A biometrical approach, third ed. McGraw hill book Co. Inc. New York.

Sulodhani Devi, E., Singh, N.B., Devi, A.B., Singh, N.G., Laishram, G.M. 2005. Gene action for fruit yield and its components in tomato (Lycopersicon esculentum Mill.). Indian Journal of Genetics. 65(3): 221-222.

Singh, N.B., Paul, A., Wani, S.H., Laishram, J.M. 2012. Heterosis Studies for Yield and its Components in Tomato (Solanum lycopersicum L.) under Valley Conditions of Manipur. International Journal of Life Sciences.1(3): 224-232.

Sunil, K.Y., Singh, B.K., Baranwal, D.K., Solankey, S.S. 2013. Genetic study of heterosis for yield and quality components in tomato (Solanum lycopersicum). African Journal of Agricultural Research.8(44): 5585-5591.

Tamta, S and Singh, J.P. 2017. Heterosis in tomato for growth and yield traits, International Journal of Vegetable Science, https://doi.org/10.1080/19315260.2017.1407857. 